\title{
QUASI REFLEXIVITY AND THE SUP OF LINEAR FUNCTIONALS
}

\author{
P.K. JaIn, K.K. Arora And D.P. Sinha
}

Quasi reflexive Banach spaces are characterised among the weakly countably determined Asplund spaces, in terms of the cardinality of the sets of linearly independent bounded linear functionals each of which does not attain its supremum on the unit sphere.

\section{INTRODUCTION}

By a well known result called the Bishop-Phelps-Bollobás theorem, the set of bounded linear functionals which attain their supremum on the unit sphere of a Banach space is norm dense in its dual. James proved that a Banach space is reflexive if and only if this set coincides with the dual space; the result was obtained for separable Banach spaces in $[6,12]$ and later for the non separable case in $[7,8,9]$.

In this paper, we shall characterise quasi reflexive Banach spaces in terms of the cardinality of the sets of linearly independent bounded linear functionals each of which does not attain its supremum on the unit sphere. However, the result is obtained for a certain class of Banach spaces $\mathcal{P}^{*}$, which we define using the concept of the projectional resolution of the identity (in short, PRI). In fact, a Banach space in $\mathcal{P}^{*}$ has a PRI of type $I$, to be defined later, such that certain complemented subspaces are also in the class $\mathcal{P}^{*}$, and weakly countably determined Asplund spaces (in particular, quasi reflexive spaces) are examples of Banach spaces in the class $\mathcal{P}^{*}$. We prove that for a positive integer $k$, a Banach space in the class $\mathcal{P}^{*}$ is quasi reflexive of order $k$ if and only if there exists a set of $k$ linearly independent functionals each of which does not attain its norm on the unit sphere and any other such set contains at most $k$ elements.

\section{Preliminaries}

In this section, we give notations and definitions required in this paper. By a functional or a projection we shall mean a bounded linear functional or projection, respectively, and a subspace will mean a closed linear subspace. For a sequence $\left\{f_{n}\right\}$, we denote by $\operatorname{conv}\left\{f_{n}\right\}$ the convex span of $\left\{f_{n}\right\}$.

Received 20 February 1996

Copyright Clearance Centre, Inc. Serial-fee code: 0004-9729/97 \$A2.00+0.00. 
We shall denote by $E, E^{*}$ and $E^{* *}$ an infinite dimensional Banach space, its first and second duals, respectively. Let $\pi: E \rightarrow E^{* *}$ be the canonical mapping. The space $E$ is said to be reflexive if $\pi(E)=E^{* *}$. James [9] proved that $E$ is reflexive if and only if every functional in $E^{*}$ attains its supremum on the unit sphere of $E$. On the other hand, Neidinger and Rosenthal [13] showed that for every non zero functional $f$ on a non reflexive Banach space $E$, there is a subspace $F$ of $E$ such that $f \mid F$ does not attain its supremum on the unit sphere of $F$.

The space $E$ is said to be quasi reflexive of order $k, 0<k<\infty$, if $\operatorname{codim}_{E^{* *}} \pi(E)=$ $k$. It is known that if $E$ is quasi reflexive of order $k$, then there is a subspace $V$ of $E^{*}$ which separates points in $E$ with $\operatorname{codim}_{E^{*}} V=k$, and an equivalent norm $\|\cdot\|_{1}$ on $E$ such that the unit ball $\left\{x \in E:\|x\|_{1} \leqslant 1\right\}$ is compact in the $\sigma(E, V)$-topology (see [2], Theorem 3.3). It follows that

$$
\begin{array}{r}
\operatorname{Codim}_{E^{* *}}\left(\pi(E) \oplus\left\{\phi \in E^{* *}: \phi(f)=0, f \in V\right\}\right) \\
=\operatorname{codim}_{E^{* *}} \pi(E)-\operatorname{codim}_{E^{*}} V=0 .
\end{array}
$$

Now, we claim that $V$ is minimal as a subspace of $E^{*}$ that separates points of $E$. For if $W$ is a proper closed subspace of $V$ which also separates points of $E$ and $f \in V$ is such that $f \notin W$, then there is a $\phi \in E^{* *}$ such that $\phi(f)=1$ and $\phi(g)=0(g \in W)$. But then there is $x \in E$ and $\phi_{0} \in E^{* *}$ with $\phi_{0}(h)=0(h \in V)$ such that $\phi=\pi(x)+\phi_{0}$, whence $\phi(g)=g(x)=0(g \in W)$. Since $W$ separates points of $E$ we have $x=0$, or equivalently, $\phi(f)=\phi_{0}(f)=0$. This is a contradiction. Thus, it follows by [15, Theorem 1] that $E$ is canonically isomorphic to $V^{*}$.

The space $E$ is called weakly compactly generated (in short, WCG) if there is a weakly compact absolutely convex set $K$ in $E$ whose linear span is dense in $E$. The concept was first considered by Amir and Lindenstrauss [1]. Every quasi reflexive Banach space is WCG and so is its dual. A weaker concept than that of WCG spaces is the concept of weakly countably determined (in short, WCD) spaces. The space $E$ is called WCD if there is a countable collection $\left\{K_{n}\right\}$ of weak ${ }^{*}$-compact subsets of $E^{* *}$ such that for each $x \in E$ and each $\phi \in E^{* *} \backslash \pi(E)$ there is an integer $n_{0}>0$ such that $x \in K_{n_{0}}$ and $\phi \notin K_{n_{0}}$. The concept of WCD Banach spaces was introduced by Vašák [17]. Every WCG Banach space is WCD and unlike WCG spaces, the subspaces of a WCD Banach space are WCD. Further, the space $E$ is said to be Asplund if every continuous convex function defined on an open set $U$ of $E$ is Frechét differentiable over a $G_{\delta}$ set dense in $U$. Every subspace of an Asplund space is Asplund and if the dual space $E^{*}$ is WCD then $E$ is Asplund (see [14]). Thus, every quasi reflexive Banach space is a WCD Asplund space.

We shall denote the cardinality of the smallest dense set in $E$ by dens $E$. Let $\omega$ denote the first ordinal with cardinality $\aleph_{0}$; the other symbols used for ordinals are 
$\alpha, \beta, \lambda$ and $\mu$. Let $\mu$ be the first ordinal with cardinality dens $E$. A long sequence $\left\{p_{\alpha}\right\}_{\omega \leqslant \alpha \leqslant \mu}$ of projections on $E$ is said to be a projectional resolution of the identity (in short, PRI) of $E$, if it satisfies the following:

$\left\|p_{\alpha}\right\|=1$

$$
\begin{array}{ll}
p_{\alpha} \cdot p_{\beta}=p_{\beta} \cdot p_{\alpha}=p_{\beta} & (\omega \leqslant \beta \leqslant \alpha \leqslant \mu) \\
\operatorname{dens} p_{\alpha}(E) \leqslant \operatorname{card} \alpha & (\omega \leqslant \alpha \leqslant \mu) \\
p_{\alpha}(E)=\overline{\bigcup\left\{p_{\beta+1}(E): \omega \leqslant \beta<\alpha\right\}} & (\omega \leqslant \alpha \leqslant \mu) \\
p_{\mu} \text { is the identity operator on } E . &
\end{array}
$$

If $E$ has a PRI $\left\{p_{\alpha}\right\}_{\omega \leqslant \alpha \leqslant \mu}$, then for each $x \in E$, the map $\alpha \rightarrow p_{\alpha}(x)$ from $[\omega, \mu]$ into $E$ is continuous and if $\beta \leqslant \mu$ is a limit ordinal then $p_{\beta}(x)=\lim _{\alpha<\beta} p_{\alpha}(x)$. Also, we shall denote by $E_{\alpha}$, the subspace $p_{\alpha}(E)$ for each $\omega \leqslant \alpha<\mu$ and note that $E_{\alpha}^{*}$ and $E_{\alpha}^{* *}$ are isomorphic to $p_{\alpha}^{*}\left(E^{*}\right)$ and $p_{\alpha}^{* *}\left(E^{* *}\right)$, respectively. The concept of PRI was introduced in [1] and a PRI in every WCG Banach space was obtained therein. For more details on PRI one may refer to [16, Chapters 19 and 20] and [3, Chapter 6].

\section{LEMMAS}

In this section we give certain intermediate results in the form of lemmas which will be required in proving the main result of the paper.

Lemma 1. Let $\left\{x_{j}\right\}$ be a sequence dense in a separable Banach space $E$ with $\operatorname{codim}_{E^{* *}}(\pi(E)) \geqslant k$ and let $\left\{\phi_{i}\right\}_{i=1}^{k} \subset E^{* *} \backslash \pi(E)$ be a linearly independent set. Then, there is $\theta$ with $0<\theta<1$ and, for each $1 \leqslant i \leqslant k$, a sequence $\left\{f_{n}^{(i)}\right\}$ in $E^{*}$ such that for each $n$
(i) $\left\|f_{n}^{(i)}\right\|<1$
(ii) $f_{n}^{(i)}\left(x_{j}\right)=0$
$(1 \leqslant j \leqslant n-1)$
(iii) $\phi_{j}\left(f_{n}^{(i)}\right)=\theta \delta_{i j}$
$(1 \leqslant j \leqslant k)$.

Proof: Assume that $\left\|\phi_{i}\right\|=1(1 \leqslant i \leqslant k)$. For each $1 \leqslant i \leqslant k$, let $V_{i}$ denote the closed linear subspace of $E^{* *}$ spanned by $\pi(E) \cup\left\{\phi_{j}\right\}_{j \neq i}$ and let $\theta$ with $0<\theta<1$ be such that $\inf _{1 \leqslant i \leqslant k} \operatorname{dist}\left(V_{i}, \phi_{i}\right)>\theta$. Let $\left\{c_{j}\right\}_{j=1}^{n+k-1}$ be scalars such that, for some $i$ with $n \leqslant i \leqslant n+k-1, c_{i}=\theta$ and $c_{j}=0$ whenever $j \neq i$. Then, for any scalars $\left\{a_{j}\right\}_{j=1}^{n+k-1}$ with $a_{i} \neq 0$, we have

$$
\left|\sum_{j=1}^{n+k-1} a_{j} c_{j}\right| \leqslant\left(\theta / \operatorname{dist}\left(V_{i}, \phi_{i}\right)\right)\left\|\sum_{j=1}^{n-1} a_{j} \pi\left(x_{j}\right)+\sum_{j=n}^{n+k-1} a_{j} \phi_{j}\right\| .
$$


By Helly's theorem, for each $1 \leqslant i \leqslant k$, each natural number $n$ and each $\varepsilon>0$ there is $f_{\varepsilon, n}^{(i)}$ in $E^{*}$ satisfying

(a) $\left\|f_{\varepsilon, n}^{(i)}\right\| \leqslant\left(\theta / \operatorname{dist}\left(V_{i}, \phi_{i}\right)\right)+\varepsilon$,

(b) $\phi_{i}\left(f_{e, n}^{(i)}\right)=\theta$,

(c) $\left(\pi\left(x_{j}\right)\right)\left(f_{e, n}^{(i)}\right)=0 \quad(1 \leqslant j \leqslant n-1)$,

(d) $\phi_{j}\left(f_{e, n}^{(i)}\right)=0 \quad(n \leqslant j \leqslant n+k-1, j \neq i)$.

Now, since $\theta / \operatorname{dist}\left(V_{i}, \phi_{i}\right)<1(1 \leqslant i \leqslant k)$, it is easy to see that, for each $1 \leqslant i \leqslant k$, and each natural number $n$, there is $f_{n}^{(i)}$ in $E^{*}$ satisfying (i)-(iii).

To make our exposition precise, we shall call a set of $k$ linearly independent functionals in $E^{*}$ each of which does not attain its supremum on the unit sphere of $E$, a James set of size $k$ in $E^{*}$.

The next lemma gives the existence of James sets of certain sizes in the dual of a separable quasi reflexive Banach space.

LEMMA 2. Let $E$ be a separable Banach space with $\operatorname{codim}_{E^{* *}} \pi(E) \geqslant k$. Then $E^{*}$ contains a James set of size $k$.

ProOF: Let $\left\{x_{j}\right\}$ be a sequence dense in $E$ and let $\left\{\phi_{i}\right\}_{i=1}^{k} \subset E^{* *} \backslash \pi(E)$ be a linearly independent set. Then, by Lemma 1 , there is $\theta$ with $0<\theta<1$ and, for each $1 \leqslant i \leqslant k$, a sequence $\left\{f_{n}^{(i)}\right\}$ in $E^{*}$, such that (i) - (iii) of Lemma 1 are satisfied. Let $1 \leqslant i \leqslant k$ be fixed. If $f \in \operatorname{conv}\left\{f_{n}^{(i)}\right\}$, then $\|f\| \geqslant \theta$ and clearly, $\lim _{n \rightarrow \infty} f_{n}^{(i)}(x)=0$ $(x \in E)$. Hence, by [9, Lemma 1], if $\left\{\lambda_{n}\right\}$ is a sequence of positive numbers with $\sum_{n=1}^{\infty} \lambda_{n}=1$, then there is a number $\alpha_{i}$ with $\theta<\alpha_{i}<1$, and a sequence $\left\{g_{n}^{(i)}\right\}$ such that

$$
\begin{aligned}
& \text { (i) } g_{n}^{(i)} \in \operatorname{conv}\left\{f_{n}^{(i)}, f_{n+1}^{(i)}, \ldots\right\}, \\
& \text { (ii) }\left\|\sum_{n=1}^{\infty} \lambda_{n} g_{n}^{(i)}\right\|=\alpha_{i}, \\
& \text { (iii) }\left\|\sum_{n=1}^{m} \lambda_{n} g_{n}^{(i)}\right\|<\alpha_{i}\left(1-\theta \sum_{n=m+1}^{\infty} \lambda_{n}\right)(m=1,2, \ldots) .
\end{aligned}
$$

Since, $\lim _{n \rightarrow \infty} f_{n}^{(i)}(x)=0(x \in E)$, it follows from (i) above that for each $x \in E$, $\lim _{n \rightarrow \infty} g_{n}^{(i)}(x)=0$; whence there is an $n_{i}$ such that $\left|g_{n}^{(i)}(x)\right|<\alpha_{i} \theta \quad\left(n \geqslant n_{i}\right)$. Now it is easy to see from (ii) and (iii) above that the functional $\sum_{n=1}^{\infty} \lambda_{n} g_{n}^{(i)}$ does not attain its supremum on the unit sphere of $E$.

Finally, the set $\left\{\sum_{n=1}^{\infty} \lambda_{n} g_{n}^{(i)}\right\}_{i=1}^{k}$ is linearly independent since, by (iii) in Lemma 
1 and (i) above, we have $\phi_{j}\left(\sum_{n=1}^{\infty} \lambda_{n} g_{n}^{(i)}\right)=\theta \delta_{i j}(1 \leqslant i, j \leqslant k)$. This completes the proof.

Let us recall that a PRI $\left\{p_{\alpha}\right\}_{\omega \leqslant \alpha \leqslant \mu}$ of $E$ is said to be of type I if

$$
\overline{\bigcup\left\{p_{\alpha}^{*}\left(E^{*}\right): \omega \leqslant \alpha<\mu\right\}}=E^{*}
$$

and shrinking if $\left\{p_{\alpha}^{*}\right\}_{\omega \leqslant \alpha \leqslant \mu}$ is a PRI of $E^{*}$ (see $[5,14]$ ). Note that a shrinking PRI is of type I. John and Zizler $[10,11]$ showed that a WCG Banach space with a WCG dual possesses a shrinking PRI. Vašák [17] proved the result in a more general case when both $E$ and $E^{*}$ are WCD. Improving upon this, Fabian [4] showed that WCD Asplund spaces admit shrinking PRI and they are WCG. For some more results on shrinking PRI one may refer to [14].

LEMma 3. Let $E$ be a Banach space with a $P R I\left\{p_{\alpha}\right\}_{\omega \leqslant \alpha \leqslant \mu}$ of type I such that $\operatorname{codim}_{E^{* *}} \pi(E) \geqslant k$. Then, there is an ordinal $\omega \leqslant \lambda<\mu$ such that $\operatorname{codim}_{E^{* *}} \pi_{\lambda}\left(E_{\lambda}\right) \geqslant$ $k$, where for each $\omega \leqslant \alpha<\mu, E_{\alpha}=p_{\alpha}(E)$ and $\pi_{\alpha}$ is the canonical map of $E_{\alpha}$ into its bidual.

Proof: First we claim that if $\phi \in E^{* *} \backslash \pi(E)$, then there is an ordinal $\omega \leqslant \lambda<\mu$ such that $p_{\lambda}^{* *} \cdot \phi \notin \pi_{\lambda}\left(E_{\lambda}\right)$. Indeed, if there is an $x \in E$ such that for each $f \in E^{*}$,

$$
\left(p_{\alpha}^{*} \cdot f\right)(x)=\phi\left(p_{\alpha}^{*} \cdot f\right) \quad(\omega \leqslant \alpha<\mu)
$$

then taking limit on $\alpha<\mu$, and as the PRI is of type I, we obtain $\phi(f)=f(x)$ $\left(f \in E^{*}\right)$, which is a contradiction.

Now, let $\left\{\phi_{i}\right\}_{i=1}^{k}$ be a linearly independent set in $E^{* *} \backslash \pi(E)$. Then, for each $1 \leqslant i \leqslant k$, there is an ordinal $\omega \leqslant \lambda_{i}<\mu$ such that $p_{\lambda_{i}}^{* *} \cdot \phi_{i} \notin \pi_{\lambda_{i}}\left(E_{\lambda_{i}}\right)$. Further, since $\left\{\phi_{i}\right\}_{i=1}^{k}$ is a linearly independent set, there is an ordinal $\omega \leqslant \lambda_{0}<\mu$ such that $\left\{p_{\lambda_{0}}^{* *} \cdot \phi_{i}\right\}_{i=1}^{k}$ is a linearly independent set. Let $\lambda=\max \left\{\lambda_{0}, \lambda_{1}, \ldots, \lambda_{k}\right\}$. Then, $\left\{p_{\lambda}^{* *} \cdot \phi_{i}\right\}_{i=1}^{k}$ is a linearly independent set such that $p_{\lambda}^{* *} . \phi_{i} \notin \pi_{\lambda}\left(E_{\lambda}\right)$ so that $\operatorname{codim} E_{I_{\lambda}^{* *} \pi_{\lambda}}\left(E_{\lambda}\right) \geqslant k$.

Before we state our next lemma we define a new class of Banach spaces containing the quasi reflexive spaces and this is the class in which we are going to look for a characterisation of quasi reflexive Banach spaces.

Definition: A Banach space $E$ is said to belong to the class $\mathcal{P}^{*}$ if it has a PRI $\left\{p_{\alpha}\right\}_{\omega \leqslant \alpha \leqslant \mu}$ of type I such that $p_{\alpha}(E)$ and $\left(p_{\alpha+1}-p_{\alpha}\right)(E)$ also belong to $\mathcal{P}^{*}$ for every $\alpha \in[\omega, \mu)$.

The above definition is along the lines of the definition of a class $\mathcal{P}$ of Banach spaces given in ([3], p.286). In fact, if the PRI in the definition of the class $\mathcal{P}$ is taken 
to be of type I, then we get the class $\mathcal{P}^{*}$. Every WCD Asplund space has a PRI of type I and its complemented subspaces are also WCD Asplund. Thus, every WCD Asplund space is in the class $\mathcal{P}^{*}$.

For a Banach space belonging to the class $\mathcal{P}^{*}$ we first prove the following.

LEMMA 4. Let $E$ be a Banach space in the class $\mathcal{P}^{*}$ such that $\operatorname{codim}_{E^{* *}} \pi(E) \geqslant$ $k$. Then, $E$ has a complemented separable subspace $F$, with a projection $p$ of $E$ onto $F$, such that $\operatorname{codim}_{F^{* *}} \pi_{p}(F) \geqslant k$, where $\pi_{p}$ is the canonical map of $F$ into $F^{* *}$.

PRoOF: Since $E$ has a PRI $\left\{p_{\alpha}\right\}_{\omega \leqslant \alpha \leqslant \mu}$ of type I with dens $E=$ card $\mu$ and with $E_{\alpha}$ and $\left(p_{\alpha+1}-p_{\alpha}\right)(E)$ belonging to $\mathcal{P}^{*}$, by Lemma 3 , there is an ordinal $\omega \leqslant \lambda<\mu$ such that $\operatorname{codim}_{E_{\lambda}^{* *}} \pi_{\lambda}\left(E_{\lambda}\right) \geqslant k$. Now, if $E_{\lambda}$ is separable, we are done. In case $E_{\lambda}$ is not separable, let $\lambda_{0}$ be the first ordinal with cardinality dens $E_{\lambda}$. Again, since $E_{\lambda}$ is in $\mathcal{P}^{*}$, let $\left\{q_{\alpha}\right\}_{\omega \leqslant \alpha \leqslant \lambda_{0}}$ be a PRI of type I of $E_{\lambda}$ such that $q_{\alpha}\left(E_{\lambda}\right)$ and $\left(q_{\alpha+1}-q_{\alpha}\right)(E)$ also belong to $\mathcal{P}^{*}$ for each $\alpha \in\left[\omega, \lambda_{0}\right)$. Another application of Lemma 3 will now give an ordinal $\omega \leqslant \beta<\lambda_{0}$ such that

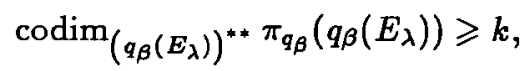

where $q_{\beta}$ is the canonical map of $q_{\beta}\left(E_{\lambda}\right)$ into its bidual. By continuing in this way, we shall obtain a complemented separable subspace of $E$ with the required property.

Now, for a bounded sequence $\left\{f_{n}\right\}$ in $E^{*}$, we use the following notation,

$$
\begin{array}{r}
L\left\{f_{n}\right\}=\left\{f \in E^{*}: \liminf _{n \rightarrow \infty} f_{n}(x) \leqslant f(x)\right. \\
\left.\leqslant \limsup _{n \rightarrow \infty} f_{n}(x), x \in E\right\} .
\end{array}
$$

Note that if $\left\{f_{n}\right\}$ is weak ${ }^{*}$-null on a subspace $F$ of $E$, then $L\left\{f_{n}\right\}$ is a subset of the annihilator of $F$ in $E^{*}$. Now, we recall the following result which is due to James [9].

Lemma 5 . Let $0<\theta<1, \lambda_{n}>0(n=1,2, \ldots)$ with $\sum_{n=1}^{\infty} \lambda_{n}=1$ and let $\left\{f_{n}\right\}$ be a sequence in the dual unit ball $B_{E^{*}}$ of a Banach space $E$ such that

$$
\|f-g\| \geqslant \theta \quad\left(f \in \operatorname{conv}\left\{f_{n}\right\}, g \in L\left\{f_{n}\right\}\right) .
$$

Then, there is $\alpha$ with $\theta \leqslant \alpha \leqslant 2$ and a sequence $\left\{g_{n}\right\}$ in $B_{E^{*}}$ with $g_{i} \in \operatorname{conv}\left\{f_{n}\right\}$ $(i=1,2, \ldots)$ satisfying
(i) $\left\|\sum_{n=1}^{\infty} \lambda_{n}\left(g_{n}-g\right)\right\|=\alpha \quad\left(g \in L\left\{g_{n}\right\}\right)$,
(ii) $\left\|\sum_{i=1}^{n} \lambda_{i}\left(g_{i}-g\right)\right\|<\alpha\left(1-\theta \sum_{i=n+1}^{\infty} \lambda_{i}\right) \quad\left(g \in L\left\{g_{n}\right\}\right)$. 


\section{MAIN RESUlt}

We are now prepared to prove the main result of this paper, wherein we characterise quasi reflexive Banach spaces among the class $\mathcal{P}^{*}$ of Banach spaces in terms of the cardinality of available James sets in the duals. The result generalises James's famous sup theorem to the quasi reflexive case.

THEOREM. Let $E$ be a Banach space belonging to the class $\mathcal{P}^{*}$. Then, $E$ is quasi reflexive of order $k$ if and only if $E^{*}$ contains a James set of size $k$ and any other James set is of size at most $k$.

Proof: Let $E$ be a quasi reflexive space of order $k$. Then, there is a subspace $V$ of $E^{*}$ which separates points in $E$ and with $\operatorname{codim}_{E^{*}} V=k$, such that $E$ is canonically isomorphic to $V^{*}$. If $f \in V$, by the Hahn-Banach theorem, there is $\psi \in V^{*}$ with $\|\psi\|=1$, whence there is $x \in E$ with $\|x\|=1$ such that $f(x)=\psi(f)=\|f\|$. Thus, it follows that any James set in $E^{*}$ is of size at most $k$. Now, if $E$ is separable and $\operatorname{codim}_{E^{* *}} \pi(E)=k$, by Lemma 2 there is a James set in $E^{*}$ of size $k$. (Note that in this case the assumption that $E$ is in $\mathcal{P}^{*}$ is redundant.) On the other hand, if $E$ is non separable, we shall construct a James set in $E^{*}$ of size $k$ as follows.

Since $E$ is in $\mathcal{P}^{*}$, by Lemma 4 , there is a complemented separable subspace $F$ of $E$ with a projection $p$ of $E$ onto $F$ such that $\operatorname{codim}_{F^{*}} \pi_{p}(F) \geqslant k$. In fact, if $\left\{\phi_{i}\right\}_{i=1}^{k}$ with $\left\|\phi_{i}\right\| \leqslant 1(1 \leqslant i \leqslant k)$ is a linearly independent set in $E^{* *} \backslash \pi(E)$, then $\mathrm{F}$ may be so chosen that $\left\{p^{* *} . \phi_{i}\right\}_{i=1}^{k}$ is a linearly independent set such that $p^{* *} . \phi_{i} \notin \pi_{p}(F)$ $(1 \leqslant i \leqslant k)$. Now let $\left\{x_{j}\right\}$ be a sequence dense in $F$. Then, as in Lemma 1 , there is $\theta$ with $0<\theta<1$ and, for each $1 \leqslant i \leqslant k$, a sequence $\left\{f_{n}^{(i)}\right\}$ in $E^{*}$ such that for each $n$
(i) $\left\|h_{n}^{(i)}\right\|<1$
(ii) $h_{n}^{(i)}\left(x_{j}\right)=0$
$(1 \leqslant j \leqslant n-1)$
(iii) $\phi_{j}\left(h_{n}^{(i)}\right)=\theta \delta_{i j}$
$(1 \leqslant j \leqslant k)$

where $h_{n}^{(i)}=p^{*} \cdot f_{n}^{(i)}(1 \leqslant i \leqslant k, n=1,2, \ldots)$.

Now, it follows from (ii) and (iii) above, that for each $1 \leqslant i \leqslant k,\left\{h_{n}^{(i)}\right\}$ is weak ${ }^{*}$ null on $F$ and

$$
\|f\| \geqslant \theta \quad\left(f \in \operatorname{conv}\left\{f_{n}^{(i)}\right\}\right) .
$$

Thus

$$
\text { (iv) } \begin{array}{r}
\|f-g\| \geqslant\left\|p^{*} \cdot(f-g)\right\|=\left\|p^{*} \cdot f\right\|=\|f\| \geqslant \theta \\
\left(f \in \operatorname{con} v\left\{h_{n}^{(i)}\right\}, g \in L\left\{h_{n}^{(i)}\right\}, 1 \leqslant i \leqslant k\right) .
\end{array}
$$

Let us denote by $W$ the subset $\pi(F) \cup\left\{\phi_{i}\right\}_{i=1}^{k}$ of $E^{* *}$. Note that since $E$ is non separable, $E^{* *}$ cannot be weak ${ }^{*}$-separable so that the linear span of $W$, being separable, is not weak ${ }^{*}$ dense in $E^{* *}$. Thus, $U=\left\{f \in E^{*}: \phi(f)=0, \phi \in W\right\}$ is a non zero 
subspace of $E^{*}$ and for each $1 \leqslant i \leqslant k, U \subset L\left\{h_{n}^{(i)}\right\}$. Further, choose a sequence $\left\{\lambda_{n}\right\}$ of scalars with $\lambda_{n}>0(n=1,2, \ldots)$ and $\sum_{n=1}^{\infty} \lambda_{n}=1$ such that there is $\delta$ with $0<\delta<\theta^{2} / 2$ and $\lambda_{n+1}<\delta \lambda_{n}(n=1,2, \ldots)$. Now, in view of (iv) above, we can apply Lemma 5 to obtain an $\alpha$ with $\theta \leqslant \alpha \leqslant 2$ and, for each $1 \leqslant i \leqslant k$, a sequence $\left\{g_{n}^{(i)}\right\}$ in the unit ball of $E^{*}$ with $g_{j}^{(i)} \in \operatorname{conv}\left\{h_{n}^{(i)}\right\}(j=1,2, \ldots)$, satisfying

$$
\begin{aligned}
& \text { (v) }\left\|\sum_{n=1}^{\infty} \lambda_{n}\left(g_{n}^{(i)}-g\right)\right\|=\alpha \quad(g \in U) \\
& \text { (vi) }\left\|\sum_{j=1}^{n} \lambda_{j}\left(g_{j}^{(i)}-g\right)\right\|<\alpha\left(1-\theta \sum_{j=n+1}^{\infty} \lambda_{j}\right) \quad(g \in U, n=1,2, \ldots) .
\end{aligned}
$$

Let us fix $i$ with $1 \leqslant i \leqslant k, x \in E$ with $\|x\|=1$ and $g \in U$ with $\|g\| \leqslant 1$. Since $\liminf _{n \rightarrow \infty} g_{n}^{(i)}(x) \leqslant g(x)$ and since $\theta \leqslant \alpha$, there exists $m$ such that

$$
\left|\left(g_{m+1}^{(i)}-g\right)(x)\right|<\theta^{2}-2 \delta \leqslant \alpha \theta-2 \delta
$$

Thus

$$
\begin{aligned}
\left|\sum_{n=1}^{\infty} \lambda_{n}\left(g_{n}^{(i)}-g\right)(x)\right| & <\left|\sum_{n=1}^{m} \lambda_{n}\left(g_{n}^{(i)}-g\right)(x)\right|+(\alpha \theta-2 \delta) \lambda_{m+1} \\
& +\left|\sum_{n=m+2}^{\infty} \lambda_{n}\left(g_{n}^{(i)}-g\right)(x)\right| \\
\leqslant & \left\|\sum_{n=1}^{m} \lambda_{n}\left(g_{n}^{(i)}-g\right)(x)\right\|+(\alpha \theta-2 \delta) \lambda_{m+1}+2\left(\sum_{n=m+2}^{\infty} \lambda_{n}\right) .
\end{aligned}
$$

Now, applying (vi) from above and since $\lambda_{n+1}<\delta \lambda_{n}(n=1,2, \ldots)$, we have

$$
\begin{aligned}
\left|\sum_{n=1}^{\infty} \lambda_{n}\left(g_{n}^{(i)}-g\right)(x)\right| & <\alpha\left(1-\theta \sum_{n=m+1}^{\infty} \lambda_{n}\right)+(\alpha \theta-2 \delta) \lambda_{m+1}+2 \delta\left(\sum_{n=m+1}^{\infty} \lambda_{n}\right) \\
& =\alpha-(\alpha \theta-2 \delta)\left(\sum_{n=m+2}^{\infty} \lambda_{n}\right)<\alpha .
\end{aligned}
$$

Hence, in view of $(v)$ above, for each $1 \leqslant i \leqslant k$ and each $g \in U, \sum_{n=1}^{\infty} \lambda_{n}\left(g_{n}^{(i)}-g\right)$ does not attain its supremum on the unit sphere of $E$.

Finally, since for each $1 \leqslant i \leqslant k, g_{n}^{(i)} \in \operatorname{conv}\left\{h_{n}^{(i)}\right\}$, it follows by (iii) above that for each $g \in U$, we have

$$
\phi_{j}\left(\sum_{n=1}^{\infty} \lambda_{n}\left(g_{n}^{(i)}-g\right)\right)=\theta \delta_{i j} \quad(1 \leqslant j \leqslant k) .
$$


Hence, for each $g \in U$, the set $\left\{\sum_{n=1}^{\infty} \lambda_{n}\left(g_{n}^{(i)}-g\right)\right\}_{i=1}^{k}$ is a James set of size $k$ in $E^{*}$. This completes the proof of the necessary part of the result.

Conversely, let $E$ be a Banach space such that the dual space contains a James set of size $k$ and any other James set is of size at most $k$. If $E$ is separable then it follows from Lemma 2 that $\operatorname{codim}_{E^{* *}} \pi(E)=k$; while if $E$ is non separable and belongs to $\mathcal{P}^{*}$ then from the above construction it follows that $E$ is quasi reflexive of order $k$.

Corollary. Let $E$ be a WCD Asplund space. Then, $E$ is quasi reflexive of order $k$ if and only if $E^{*}$ contains a James set of size $k$ and the size of any other James set in $E^{*}$ is at most $k$.

The assertion of the theorem answers positively the problem of extending James theorem to the quasi reflexive case in the particular class $\mathcal{P}^{*}$. The problem remains open whether one can prove the same without the assumption in the definition of $\mathcal{P}^{*}$. In fact, the main hindrance is going to come in obtaining the assertion of Lemma 4 without the extra assumption. However, if not true in the general case, it may be true for a class larger than $\mathcal{P}^{*}$ or for that matter for a different class altogether containing the quasi reflexive Banach spaces.

\section{REFERENCES}

[1] D. Amir and J. Lindenstrauss, 'The structure of weakly compact sets in Banach spaces', Ann. of Math. 88 (1968), 35-46.

[2] P. Civin and B. Yood, 'Quasi-reflexive spaces', Proc. Amer. Math. Soc. 8 (1957), 906-911.

[3] R. Deville, G. Godefroy and V. Zizler, Smoothness and renormings in Banach spaces (Longman Scientific and Technical, Harlow, 1993).

[4] M. Fabian, 'Each weakly countably determined Asplund space admits a Frechét differentiable norm', Bull. Austral. Math. Soc. 36 (1987), 367-374.

[5] P.K. Jain, K.K. Arora and D.P. Sinha, 'Weak' sequential compactness of dual unit balls of Banach spaces with PRI', J. Indian. Math. Soc. (to appear).

[6] R.C. James, 'Reflexivity and the supremum of linear functionals', Ann. Math. 66 (1957), 159-169.

[7] R.C. James, 'Characterizations of reflexivity', Studia Math. 23 (1964), 205-216.

[8] R.C. James, 'Weakly compact sets', Trans. Amer. Math. Soc. 113 (1964), 129-140.

[9] R.C. James, 'Reflexivity and the sup of linear functionals', Israel J. Math. 13 (1972), 289-300.

[10] K. John and V. Zizler, 'Duals of Banach spaces which admit non trivial smooth functions', Bull. Austral. Math. Soc. 11 (1974), 161-166.

[11] K. John and V. Zizler, 'Weak compact generating in duality', Studia Math. 55 (1976), 1-20.

[12] V. Klee, 'Some characterizations of reflexivity', Rev. Cienc. (Lima) 52 (1950), 15-23. 
[13] R. Neidinger and H.P. Rosenthal, 'Norm - attainment of linear functionals on subspaces and characterization of Tauberian operators', Pacific J. Math. 118 (1985), 215-228.

[14] J. Orihuela and M. Valdivia, 'Projective generators and resolutions of identity in Banach spaces', Rev. Mate. Univ. Comp. Madrid 2 (1989), 179-199.

[15] A.F. Ruston, 'Conjugate Banach spaces', Proc. Camb. Phil. Soc. 53 (1957), 576-580.

[16] I. Singer, Bases in Banach spaces II (Springer-Verlag, Berlin, Heidelberg, New York, 1981).

[17] L. Vašák, 'On one generalization of weakly compactly generated Banach spaces', Studia Math. 70 (1981), 11-19.

Department of Mathematics

University of Delhi

Delhi 110007

India

Department of Mathematics

Dyal Singh College

University of Delhi

Lodi Road

New Delhi 110003

India

\author{
Department of Mathematics \\ Rajdhani College \\ University of Delhi \\ Ring Road, Raja Garden \\ New Delhi 110015 \\ India
}

